\title{
Two Bone Fragment Manipulation in Computer-Assisted Preoperative Planning: Restoration of the Radial Bow
}

\author{
G.S. Athwal ${ }^{1}$, S. Leclaire $^{2}$, R.E. Ellis ${ }^{1}$, and D.R. Pichora ${ }^{1}$ \\ ${ }^{1}$ Division of Orthopædics, Department of Surgery \\ ${ }^{2}$ School of Computing \\ Queen's University at Kingston, Canada K7L 3N6
}

\begin{abstract}
Distal radius fractures are the most common fracture seen in hospital emergency departments. While most patients have a good outcome, a small percentage of fractures heal in a displaced position (malunion), which may cause pain, stiffness, and weakness of the wrist and forearm. We have developed a computerassisted technique for correction (osteotomy) of distal radius malunion. Outcome studies have shown that this procedure is accurate, reliable, and safe. It has advantages over a computer-assisted conventional technique, including less fluoroscopic radiation to the patient and surgical team, and a quicker learning curve for nonexpert surgeons. Our initial technique corrected only the initial fragment of the radius, leaving the proximal radius unchanged (relative to the ulna which is the fixed uninvolved element in the forearm in these cases). A new development reported here is the incorporation of correction of the proximal radius. This allows for full correction of the radial ulnar convergence, restoring the bow of the radius. We noted improved fixation plate positioning and full forearm rotation post operatively.
\end{abstract}

\section{Objectives}

To improve correction of the radial bow in computer-assisted reconstruction of the malunited distal radius.

\section{Background}

Malunions of the distal radius may cause alterations in the alignment, kinematics and load transfer across the wrist. They have classically been described as complex multidirectional deformities referenced from the static proximal radius. We hypothesize that the proximal radius fragment contributes to the global deformity of malunion in convergence with the ulna. This complex deformity results in a decrease in the radial bow. The radial bow has been described as the major lateral convex curve of the middle three-fifths of the radius [2]. Schemitsch and Richards have shown a relationship between the restoration of the normal radial bow and functional indices such as grip strength and rotation of the forearm [1]. 


\section{Design and Methods}

Two manually active patients with symptomatic distal radius malunions underwent CTbased computer-assisted distal radius osteotomy [3]. During the pre-operative surgical planning phase it was noted that the proximal radius contributed to the global deformity of the radius. Using a custom pre-operative surgical planner a virtual osteotomy was conducted and both the proximal and distal fragment were independently manipulated to recreate the anatomy of the distal radius and to correct the radial bow. Our æfixationbasedÆ method of computer-assisted distal radius osteotomy incorporates the fixation plate and the 3D location of key screw holes in the surgical plan.

\section{Results and Conclusions}

Radial inclination, ulnar variance and volar tilt were anatomically restored in both cases. The maximum radial bow and the location of the maximum radial bow were anatomically restored. Computer-assisted planning allows the correction of key wrist parameters (radial inclination, ulnar variance and volar tilt) and the appreciation and restoration of complex malalignments such as the radial bow. We have found that in a 2-bone limb segment, such as the forearm, it is necessary to correct the deformity in both ends of the affected bone (relative to the intact ulna and the fixation plate) in order to fully restore the anatomy. To our knowledge this is the first description of a computer-assisted preoperative planning system that allows complete correction of a deformity by correcting the deformities at both ends of a malunited bone relative to the intact normal bone in that limb segment.

Acknowledgments. This research was supported in part by the Institute for Robotics and Intelligent Systems, the Ontario Research and Development Challenge Fund, and the Natural Sciences and Engineering Research Council of Canada.

\section{References}

1. E. H. Schemitsch and R. R. Richards. The effect of malunion on functional outcome after plate fixation of fractures of both bones of the forearm in adults. Journal of Bone and Joint Surgery, 74A(7):1068-1078.

2. R. R. Tarr, A. I. Garfinkel, and A. Sarmiento. The effects of angular and rotational deformities of both bones of the forearm. Journal of Bone and Joint Surgery, 66A:65-70.

3. H. Croitoru, R. E. Ellis, C. F. Small, R. Prihar, and D. R. Pichora. Fixation-based surgery: A new technique for distal radius osteotomy. Journal of Computer Aided Surgery, 6:160-169, 2001. 